\title{
Artifacts Removal in Melanoma Using Various Preprocessing Filters
}

\author{
R. Ramya Ravi ${ }^{{ }^{*}}$, R.S. Vinod Kumar ${ }^{2}$, N. Shanila ${ }^{3}$ \\ ${ }^{l}$ Research Scholar, Department of Electronics \& Communication Engineering, Noorul Islam Centre for Higher Education, Tamil Nadu, \\ India. \\ ${ }^{2}$ Professor, Department of Electronics \& Communication Engineering, Noorul Islam Centre for Higher Education, Kumaracoil, Tamil \\ Nadu, India. E-mail:rsvinodkumar69@yahoo.co.in \\ ${ }^{3}$ Research Scholar, Department of Electronics \& Communication Engineering, Noorul Islam Centre for Higher Education, Tamil Nadu, \\ India.E-mail:shanilaabin@gmail.com \\ *Corresponding author E-mail:ramyaravir03@gmail.com
}

\begin{abstract}
Preprocessing plays an important role for artifacts removal and quality improvement. In this paper, noise and hair removal techniques for melanoma in dermoscopic images is proposed. Here the performance of four filters for noise removal namely Wiener, Mean, Median, and Gaussian Filters are studied. Of these the performance of Gaussian filter is proved to be best. In addition Morphological Filter is used for hair removal. The noise and hair removal filtering processes help to enhance the quality of the image and thus aid to improve the segmentation results. The performance of the preprocessing filters is compared using quantifying parameters like MSE, PSNR, and SSIM.
\end{abstract}

Keywords: Preprocessing, morphological filter, gaussian filter, median filter, mean filter, wiener filter, melanoma.

\section{Introduction}

Melanoma is the type of skin cancer that develops from the pigment cells known as melanocytes. The only way to reduce the death rate is the early detection of the disease. Malignant melanoma is a serious type of skin cancer and is also found in the bowel and the eye. It occurs due to the uncontrolled growth of pigment cells called melanocytes. The steps included in melanoma diagnosis are Preprocessing, Segmentation, Feature Extraction and Classification. All these steps play an important role in melanoma diagnosis. The artifacts which affect the interested area during segmentation are filtered using various filters. During preprocessing the quality of the image is also get upgraded.

The organisation of remaining paper is as follows. Section 2 deals with the related works in pre-processing. While Section 3 briefs the proposed techniques, Section 4 discusses about the experimental results and the performance comparison of the proposed work. The Conclusion and Future work are given in Section 5 .

\section{Related Works}

Melanoma is the fatal form of skin cancer, only early detection reduces death in patients. The artifacts removal in melanoma is a tedious process in melanoma detection. In pre-processing the artifacts are eliminated using proposed techniques. Major artifacts removal includes noise removal and hair removal, which can enhance the quality of the image.
Even though a good volume of research is carried out in the area of pre-processingof melanoma for the past two decades, there are chances of many new researches in this area.

Some of thepre-processing techniques for noise and hair removal which are closely related to the proposed work are listed below. Some of the works done in noise removal follows.

Leo Grady et al., [2006] introduced anisotropic diffusion for image filtering. Simon Perreault et al., [2007] proposed median filtering for high resolution medical images.Emre Celebi et al., [2009] removed artifacts using filters such as the Gaussian filter (GF), median filter(MF), or anisotropic diffusion filters (ADF). Maryam Sadeghi et al., [2011] proposed high pass filter for eliminating the low frequency noise.

A few works carried out in hair removal are Lee T et al., [1997] presented a new method to remove thick hairs called Dull Razor. This pre-processing step empowered the segmentation program to achieve satisfactory results. Do Hyun Chunget et al., [2000] used PDE-based continuous morphological filter to remove unwanted hairs. Harald Ganster et al., [2001] excluded the hair artifacts using grey-scale morphological operation of closing. Silveira et al., [2009] introduced morphological closing filter and median filter to eradicate features from dark hair. Paul Wighton et.al [2011] suggested gray scale morphological closing operation with a linear structuring element to spot dark hair. Qaisar et al., [2011] introduced hair repairing algorithm for the removal and restoration of hair. Qaiser et al., [2011] used Homomorphic filtering for specular reflection reduction, air bubbles or dermoscopic gel reduction they used adaptive and recursive weighted median filter for hair and line reduction he proposed artifacts removal algorithm. Abuzaghleh, O et al., [2015] introduced 84 directional filters to identify and ignore hair in the lesion. Reda Kasmi etal., [2016] presented a new approach to reduce the impact of bubbles 
using $11 \times 11$ median filters for thin hairs and Gabor filter for thick hairs. Andrea Pennisia et al., [2016] processed through a morpho184 logical transformation in order to remove hair, while preserving the visual properties of the lesion region. Fengying Xie et al.,[2017] obtained partial differential equation (PDE)-based image repair method for the detection and removal of hairs from dermoscopic images. In this section a brief discussion of noise and hair removal techniques for melanoma were done.

\section{Proposed Work}

Artifacts elimination is an important task in melanoma detection. Hair, noise, air bubbles are the artifacts eliminated by this process. Elimination of these artifacts is very essential before melanoma segmentation. Therefore pre-processing plays an important role in melanoma segmentation. In this paper artifacts removal is carried out in two steps namely, one is noise removal and other one is hair removal to enrich the quality of the image. For noise removal four filters are used, namely Wiener, Mean, Median, and Gaussian filters and Morphological filter are used for hair removal. The preprocessed output is then given to the input of segmentation process. The performances of Wiener, Mean, Median, and Gaussian filter are compared in this paper. The explanation of the following filters is given below.

Mean filter is also known as average filter. Smoothening of image was done by this filter. The filter works as a low-pass filter. Median filter is used to remove noise from an image. It plays an excellent role in preprocessing by preserving the edge of an image while removing noise. Wiener filter removes the additive noise and inverts the blurring simultaneously. It gives minimum mean squared error of an image. The Gaussian filter is a type of imageblurring filter that uses a Gaussian function for calculating the transformation to apply to each pixel in the image. The performance of a Gaussian function in one dimension is given in Equation (1). The Gaussian filter is used for noise removal.

$G(x)=\frac{1}{\sqrt{2 \pi} \sigma^{2}} e^{-\frac{x^{2}}{2 \sigma^{2}}}$

Determining the most efficient methods, reduce the rate of errors. It is a vital issue among researchers. Morphological operation consists of four operations, dilation, erosion, opening and closing. The process of performance that depends on shapes is known as Morphology. Dilation operator is used to enhance pixels and the erosion operator is used to eliminate pixels. In this paper for hair removal morphological filter are used.

The various steps involved in the pre-processing operation are listed below.

1. Select an input image from the dataset.

2. Perform filtering operation using Wiener, Mean, Median, and Gaussian Filters for noise removal.

3. Perform filtering using Morphological Filter for hair removal.

4. Calculate the quantifying parameters MSE, PSNR and SSIM.

5. Repeat the steps 2,3 , and 4 for the remaining images.

\section{Experimental Results and Discussion}

In Melanoma preprocessing artifacts removal plays an important role. The simulations were performed using MATLAB R2014a in Intel(R) Core(TM) 2 Duo CPU T6570 @ 2.10GHz, 2:00GB RAM and 64 bit Operating System. Preprocessing is done using five filters viz., Wiener, Mean, Median, Gaussian and Morphological Filter. Figs. 1 and 2 show the output images of various filters for IMD002 and IMD009, in which (a) is the input image,(b) is the output of wiener Filter, (c) is the output of Mean Filter, (d) is the output of Median Filter, (e) is the output of Gaussian Filter and (f) is the output of the Morphological Filter. Comparing the four filtered images it is clear that the output of the Gaussian Filter was proved to be best for noise removal and Morphological Filter is more suitable for hair removal.

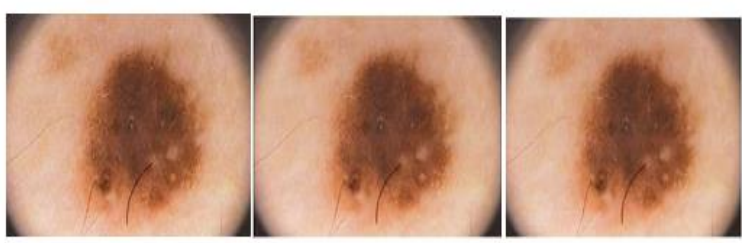

(a)

(b)

(c)

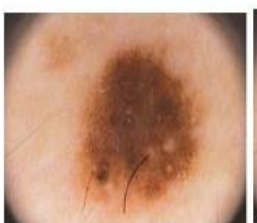

(d)

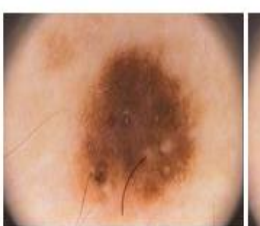

(e)

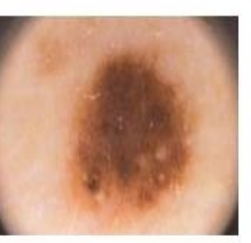

(f)
Fig. 1: Outputs of various filters for IMD002. a) input image b) wiener filter c) mean filter d) median filter e) gaussian filter f) morphological filter

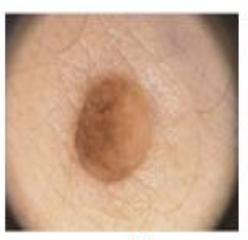

(a)

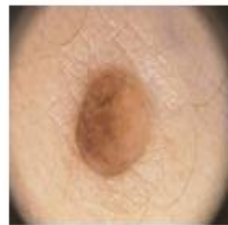

(d)

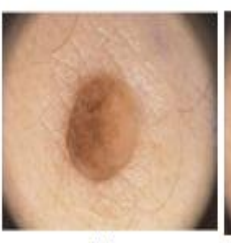

(b)

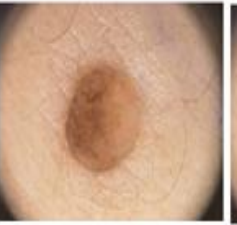

(e)

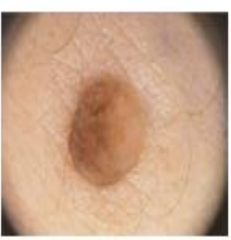

(c)

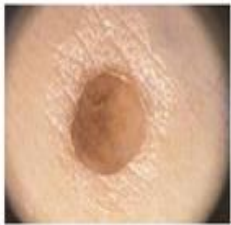

Fig. 2: Outputs of various filters for IMD009. a) input image b) wiener filter c) mean filter d) median filter e) gaussian filter f) morphological filter

To compare the various image pre-processing techniques, three error metrics are used namely Mean Squared Error (MSE), Peak Signal-to-Noise Ratio (PSNR) and Structural Similarity Index (SSIM).The MSE is the cumulative squared error between the compressed and the original image, which is given by Equation (2)

$\mathrm{MSE}=\frac{1}{\mathrm{ab}} \sum_{\mathrm{x}=0}^{\mathrm{b}-1} \sum_{\mathrm{y}=0}^{\mathrm{a}-1}[\mathrm{M}(\mathrm{x}, \mathrm{y})-\mathrm{N}(\mathrm{x}, \mathrm{y})]^{2}$

where $\mathrm{M}(\mathrm{x}, \mathrm{y})$ is the original image, $\mathrm{N}(\mathrm{x}, \mathrm{y})$ is the approximated version (decompressed image) and $a, b$ are the dimensions of the images. Peak Signal to Noise Ratio (PSNR) is inversely proportional to MSE. It is defined as the ratio of quality measurement between the original image and compressed image. For better compressed or reconstructed image the PSNR value is high. The expression for PSNR is given in Equation (3)

PSNR $=10 \log _{10}\left(\frac{\mathrm{P}^{2}}{\mathrm{MSE}}\right)$

where $P$ is the maximum fluctuation in the input image.

Structural Similarity Index Measure (SSIM) is also a quantifying parameter used for measuring image quality. Similarity between original and compressed images can be determined using this SSIM parameter and is given in Equation (4)

$\operatorname{SSIM}(\mathrm{x}, \mathrm{y})=\frac{\left(2 \mu_{\mathrm{x}} \mu_{\mathrm{y}}+\mathrm{C}_{1}\right)\left(2 \sigma_{\mathrm{xy}}+\mathrm{C}_{2}\right)}{\left(\mu_{\mathrm{x}}^{2}+\mu_{\mathrm{y}}^{2}+\mathrm{C}_{1}\right)\left(\sigma_{\mathrm{x}}^{2}+\sigma_{\mathrm{y}}^{2}+\mathrm{C}_{2}\right)}$ 
For SSIM index the numerical value lies between -1 and 1 . For identical images numerical value is always 1.The quantifying parameters are calculated. Experiments are done for 25 samples and tabulation is done for 10 samples outputs. $\mathrm{PH} 2$ database is used in the proposed work, which contains a set of 200 dermoscopic images and its binary mask. The quantifying parameters are tabulated in Table 1.

Table 1: Performance comparison table of MSE, PSNR and SSIM for various filters

\begin{tabular}{|c|c|c|c|c|c|c|c|c|c|c|c|c|}
\hline \multirow[t]{2}{*}{ IMAGES } & \multicolumn{3}{|c|}{ WIENER FILTER } & \multicolumn{3}{|c|}{ MEAN FILTER } & \multicolumn{3}{|c|}{ MEDIANFILTER } & \multicolumn{3}{|c|}{ GAUSSIANFILTER } \\
\hline & MSE & $\begin{array}{l}\text { PSNR } \\
\text { dB }\end{array}$ & SSIM & MSE & $\begin{array}{l}\text { PSNR } \\
\mathrm{dB}\end{array}$ & SSIM & MSE & $\begin{array}{l}\text { PSNR } \\
\mathrm{dB}\end{array}$ & SSIM & MSE & PSNR dB & SSIM \\
\hline IMD002 & 9.245 & 38.472 & 0.856 & 9.245 & 38.472 & 0.856 & 7.974 & 39.114 & 0.854 & 2.736 & 43.759 & 0.856 \\
\hline IMD006 & 7.023 & 39.666 & 0.898 & 7.023 & 39.666 & 0.898 & 5.235 & 40.941 & 0.897 & 2.100 & 44.907 & 0.898 \\
\hline IMD016 & 9.142 & 38.521 & 0.898 & 9.142 & 38.521 & 0.898 & 5.945 & 40.389 & 0.905 & 3.169 & 43.121 & 0.898 \\
\hline IMD017 & 8.052 & 39.072 & 0.881 & 8.052 & 39.072 & 0.881 & 6.395 & 40.073 & 0.882 & 2.259 & 44.591 & 0.881 \\
\hline IMD023 & 12.134 & 37.291 & 0.871 & 12.134 & 37.291 & 0.871 & 9.301 & 38.445 & 0.875 & 2.883 & 43.532 & 0.871 \\
\hline IMD033 & 10.893 & 37.759 & 0.853 & 10.893 & 37.759 & 0.853 & 3.214 & 38.644 & 0.857 & 3.214 & 43.061 & 0.853 \\
\hline IMD133 & 8.569 & 38.801 & 0.839 & 8.569 & 38.801 & 0.839 & 7.357 & 39.464 & 0.834 & 2.181 & 44.743 & 0.839 \\
\hline IMD199 & 10.460 & 37.936 & 0.853 & 10.461 & 37.936 & 0.853 & 8.594 & 38.789 & 0.856 & 2.536 & 44.089 & 0.853 \\
\hline IMD434 & 12.295 & 37.234 & 0.831 & 12.295 & 37.234 & 0.831 & 8.594 & 38.651 & 0.831 & 2.967 & 43.408 & 0.831 \\
\hline IMD437 & 12.489 & 37.165 & 0.839 & 12.489 & 37.165 & 0.839 & 8.594 & 39.464 & 0.856 & 3.306 & 42.937 & 0.839 \\
\hline
\end{tabular}

From Table 1 it is understood that MSE value is small and PSNR value is large for Gaussian Filters. Therefore Gaussian Filter is preferred to remove noise in Melanoma. Figs. 3, 4, and 5 show the graphical representation of the three quantifying parameters viz. MSE, PSNR, and SSIM for the four filters, which show that the performance of Gaussian Filter is better than the others three filters. In the next category i.e., the removal of hair, the performance of Morphological filter is found to be the best.[16]

\section{Images vs MSE}

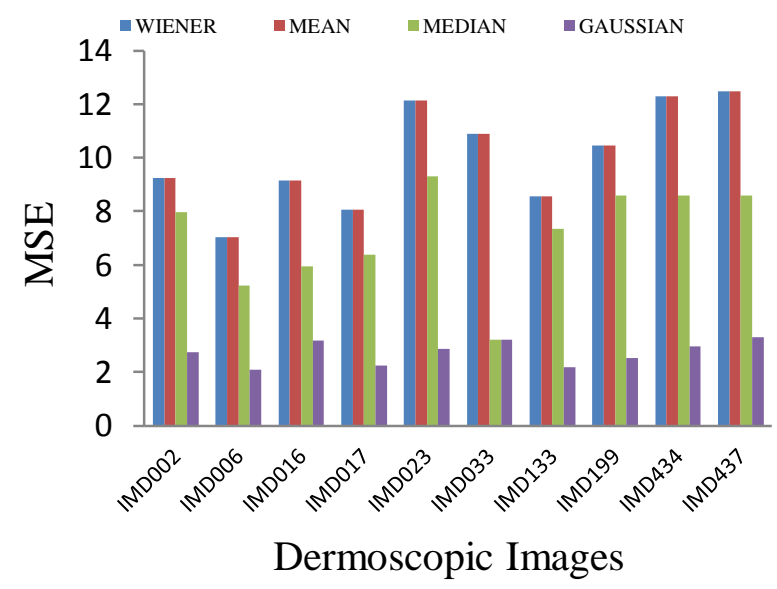

Fig. 3: Graphical representation of images vs MSE

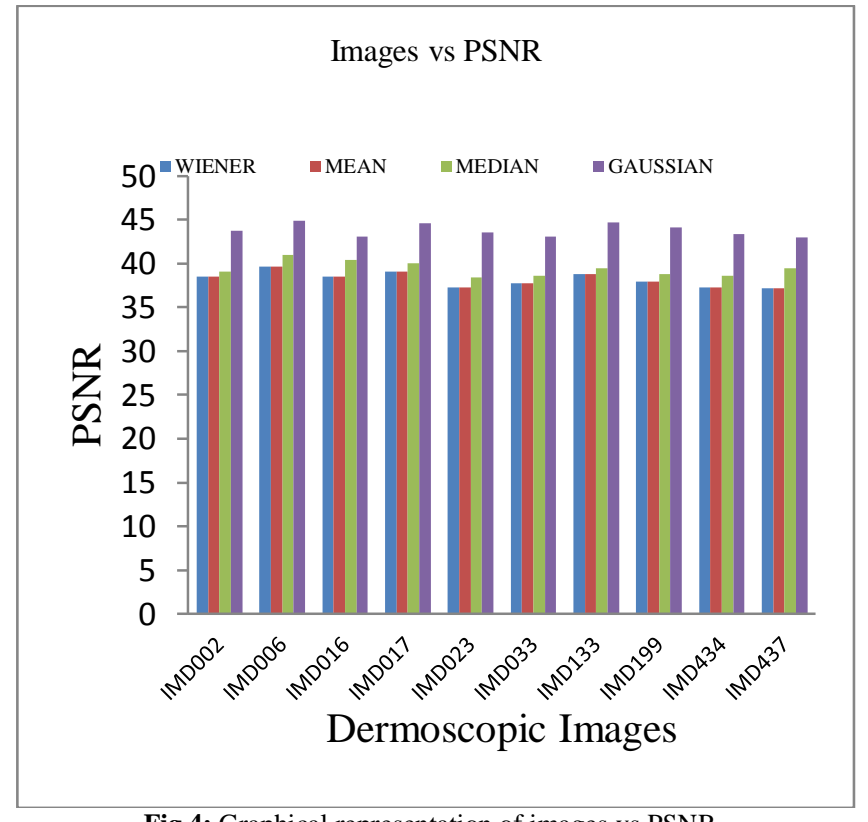

Fig.4: Graphical representation of images vs PSNR

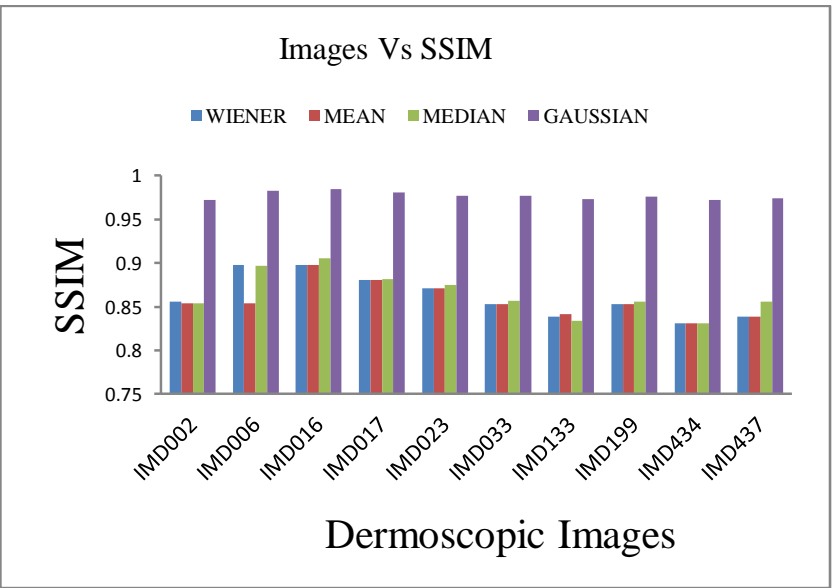

Fig. 5: Graphical representation of images vs. SSIM

The performance of the proposed filters are compared graphically using the three quantifying parameters namely MSE, PSNR, and SSIM as shown in Figs 3 to 5. The graphical representation also confirm that the performance of the Gaussian Filter is best suited for noise removal in dermoscopic images.[17] 


\section{Conclusion}

The performance of five pre-processing filters used for noise removal and hair removal in melanoma were discussed and compared in this paper. Four filters were used for noise removal. Of them Gaussian Filter is found to be more suitable for noise removal in dermoscopic images. The performance of Morphological Filter is found to be satisfactory for hair removal. In this paper, noise and hair removal techniques are only proposed. In future, the work can be extended by introducing a contrast enhancement techniques should be proposed to improve the quality of the dermoscopic images.

\section{References}

[1] Lee T, Ng V, Gallagher R, Coldman A \& McLean D, "Dullrazor: A software approach to hair removal from images", Computers in biology and medicine, Vol.27, No.6,(1997), pp.533-543.

[2] Chung DH \& Sapiro G, "Segmenting skin lesions with partialdifferential-equations-based image processing algorithms", IEEE transactions on Medical Imaging, Vol.19, No.7,(2000),pp.763-767.

[3] Ganster H, Pinz P, Rohrer R, Wildling E, Binder M \& Kittler H, "Automated melanoma recognition", IEEE transactions on medical imaging, Vol.20, No.3, (2001), pp.233-239.

[4] Grady L, "Random walks for image segmentation", IEEE transactions on pattern analysis and machine intelligence, Vol.28, No.11, (2006), pp.1768-1783.

[5] Perreault S \& Hébert P, "Median filtering in constant time", IEEE transactions on image processing, Vol.16, No.9,(2007), pp.23892394.

[6] Silveira M, Nascimento JC, Marques JS, Marçal AR, Mendonça T, Yamauchi S, Maeda J \& Rozeira J, "Comparison of segmentation methods for melanoma diagnosis in dermoscopy images", IEEE Journal of Selected Topics in Signal Processing, Vol.3, No.1,(2009), pp.35-45.

[7] Celebi ME, Iyatomi H, Schaefer G \& Stoecker WV, "Lesion border detection in dermoscopy images", Computerized medical imaging and graphics, Vol.33, No.2,(2009), pp.148-153

[8] Sadeghi M, Razmara M, Lee TK \& Atkins MS, "A novel method for detection of pigment network in dermoscopic images using graphs", Computerized Medical Imaging and Graphics, Vol.35, No.2,(2011), pp.137-143

[9] Wighton P, Lee TK, Lui H, McLean DI \& Atkins MS, "Generalizing common tasks in automated skin lesion diagnosis", IEEE Transactions on Information Technology in Biomedicine, Vol.15, No.4,(2011), pp.622-629.

[10] Abbas Q, Celebi ME \& García IF, "Hair removal methods: a comparative study for dermoscopy images", Biomedical Signal Processing and Control, Vol.6, No.4,(2011), pp.395-404.

[11] Abbas Q, Fondón I \& Rashid M, "Unsupervised skin lesions border detection via two-dimensional image analysis", Computer methods and programs in biomedicine, Vol.104, No.3,(2011), pp.e1-e15.

[12] Abuzaghleh O, Barkana BD \& Faezipour M, "Noninvasive realtime automated skin lesion analysis system for melanoma early detection and prevention", IEEE journal of translational engineering in health and medicine, Vol.3, (2015), pp.1-12.

[13] Kasmi R \& Mokrani K, "Classification of malignant melanoma and benign skin lesions: implementation of automatic ABCD rule", IET Image Processing, Vol.10, No.6,(2016), pp.448-455.

[14] Pennisi A, Bloisi DD, Nardi D, Giampetruzzi AR, Mondino C \& Facchiano A, "Skin lesion image segmentation using Delaunay Triangulation for melanoma detection", Computerized Medical Imaging and Graphics, Vol.52, (2016), pp.89-103.

[15] Xie F, Fan H, Li Y, Jiang Z, Meng R \& Bovik A, "Melanoma classification on dermoscopy images using a neural network ensemble model", IEEE transactions on medical imaging, Vol.36, No.3, (2017), pp.849-858.

[16] Z Iskakova, M Sarsembayev, Z Kakenova (2018). Can Central Asia be integrated as asean? Opción, Año 33. 152-169.

[17] G Cely Galindo (2017) Del Prometeo griego al de la era-biós de la tecnociencia. Reflexiones bioéticas Opción, Año 33, No. 82 (2017):114-133 